\title{
THE EGSYS AND OESIL RISK SCORES FOR CLASSIFICATION OF CARDIAC ETIOLOGY OF SYNCOPE: COMPARISON, REVALUATION, AND CLINICAL IMPLICATIONS
}

\author{
Jiri Plasek $^{\mathrm{a}}$, Vlastimil Doupal ${ }^{\mathrm{b}}$, Jana Fürstova ${ }^{\mathrm{c}}$, Arnost Martinek ${ }^{\mathrm{a}}$ \\ a Department of Internal Medicine, University Hospital and Faculty of Health Studies, University of Ostrava, Czech Republic \\ ${ }^{b}$ Department of Cardiology, University Hospital and Faculty of Medicine and Dentistry, Palacky University Olomouc, Czech \\ Republic \\ c Institute of Computer Science, Academy of Sciences of the Czech Republic, Prague, Czech Republic \\ E-mail:jiri_plasek@centrum.cz
}

Received: August 14, 2009; Accepted: March 17, 2010

Key words: Syncope/Cardiac Syncope/EGSYS/OESIL/Emergency Department/Decision-Making/Syncope Management Unit.

Aims. EGSYS-U, EGSYS-M, and OESIL risk scores for syncope evaluation are introduced, evaluated retrospectively, and their ability to classify cardiac syncopes is compared. The specificity and sensitivity of the scores were analyzed. Guidelines for the practical application of the scoring systems are provided to reduce unnecessary hospital admissions.

Methods. Retrospectively, 153 patients with syncope admitted to the Faculty Hospital in Ostrava in the year 2008 were enrolled for scoring with the EGSYS and OESIL risk scores. The computed scores were assessed with respect to the diagnosed etiology of the syncope.

Results. For each score system, the hypothesis that there is no difference in the score between the cardiac and the non-cardiac group was rejected (all p-values below 0.0001). Strong correlation between the three score systems was shown (Spearman correlation coefficient: $r=0.71 p<0.0001$ for EGSYS-U and OESIL, $r=0.88 p<0.0001$ for EGSYS-U and EGSYS-M, $r=0.60 \mathrm{p}<0.0001$ for EGSYS-M and OESIL). Sensitivity and specificity of the scores were computed and compared.

Conclusions. All three score systems were found capable of classifying cardiac syncopes, reducing unnecessary hospital admissions and improving syncope risk evaluation. EGSYS-U was recommended for emergency syncope management because it was found to have both high sensitivity and high specificity.

\section{INTRODUCTION}

According to the European Society of Cardiology (ESC) Guidelines ${ }^{1}$, syncope is a symptom, defined as a transient, self-limited loss of consciousness and postural tone. The onset of syncope is relatively rapid, and the subsequent recovery is spontaneous, complete, and usually prompt. The underlying mechanism is in principle a transient global cerebral hypoperfusion of varying etiology. The above combination is the basis for differentiating a syncope from other non-syncopal transient loss of consciousness attacks (TLOC) including cataplexy, seizures, drop attacks and even simple falls.

Syncope represents a common clinical problem. The extension of syncope as a public health problem has been documented since the Framingham Study Report ${ }^{2}$ showed the incidence of self-reported syncope to be 6.2 per 1000 person-years with a cumulative incidence of approximately $6 \%$ over 10 years. It has been reported ${ }^{2-6}$ that syncope accounts for about $5 \%$ of Emergency Department (ED) visits and in the age group of patients older than 65 , syncope becomes the sixth most common cause of hospitalization. Shen et al..$^{5}$ report that in selected patient popu- lations, the lifetime prevalence of syncope reaches almost $50 \%$. We are not aware of any published data concerning the prevalence of syncope among the Czech population.

Syncopes can be classified ${ }^{1}$ according to the principal known causes of the loss of consciousness into the following five categories: Neurally-mediated, Orthostatic hypotension caused, Cardiac arrhythmia caused, Structural cardiac or cardiopulmonary disease caused, Cerebrovascular. It is known ${ }^{2}$ that persons with cardiac syncope are at increased risk of death, while vasovagal syncope appears to have a benign prognosis. In this article, we shall therefore adopt a simpler classification, and distinguish between cardiac and non-cardiac syncopes only.

Investigation of the cause of a syncope may be expensive, extensive (telemetry monitoring, echocardigraphy, exercise ECG, signal averaged ECG, head-up-tilt test), invasive (electro-physiological study, coronarography in the case of coronary artery disease suspicion), and often not accurate ${ }^{7}$. Although the cause may eventually be discovered by a specialist, from the ED point of view, it is essential to distinguish patients with cardiac syncopes, who should be admitted to the hospital, from those with non-cardiac syncopes, who can mostly be treated in outpatients. 
The introduction of syncope management units (SMUs) is a possible strategy to reduce unnecessary hospital admissions. A SMU is a specialized unit in the Emergency Department (including its own staff) designated for syncope evaluation on a multidisciplinary basis, including telemetry monitoring, echocardiography, tilttable test and electrophysiological study equipment and risk prediction rules. The SMUs have been shown to be cost-effective and preserving high quality care, both in North American and European studies ${ }^{3,5}$.

Unfortunately, establishing and maintaining SMUs is beyond the capacity of most community hospitals around the world (there is no SMU in the Czech Republic). However, instead of SMUs, simple ED decision-making strategies can be used. These strategies are based on risk stratification rules combining anamnestic and basic clinical data. Several such rules were proposed recently, e.g. the OESIL risk score ${ }^{6}$, the EGSYS risk score ${ }^{3}$ and the San Francisco Syncope Rule ${ }^{8}$. Surprisingly, the adherence to these easy syncope stratification methods seems to be low, possibly due to lack of leadership, lack of convincing data based on randomized clinical studies, and lack of a widely accepted standardized approach. We are not aware that any ED of any Czech hospital uses these score systems.

In this study, we use the EGSYS and OESIL risk scores, which originated as a result of the Italian Syncope Management Unit project ${ }^{9}$. These systems were designed to simplify hospitalization decision, through distinguishing between cardiac and non-cardiac syncopes. By means of uni- and multi-variate logistic regression, the most important independent predictors of a cardiac syncope were identified in a list of 52-item formulary. According to the value of the regression coefficient, positive or negative weights were associated to the respective predictors ${ }^{3,9,10}$. The OESIL score ${ }^{6}$ is based on mortality data and it is designed to be as simple as possible (see Appendix 1).

There have been many attempts to select anamnestic risk factors or to select specific markers of vasovagal syncope, which is the most common type of a non-cardiac syncope. One of the best known choices was made by Calkins et al. ${ }^{11}$, who identified anamnestic features able to distinguish between AV block, ventricular tachycardia and vasovagal syncope. However, the result was not a point score but a questionnaire. Another approach ${ }^{7}$ to differentiate serious (cardiac) syncopes from vasovagal ones focuses on vasovagal syncope itself, constructing a score system represented by a yes-no questionnaire with an overall accuracy of $90 \%$, sensitivity of $89 \%$ and specificity of $91 \%$ (ref. ${ }^{7}$ ). Its main disadvantage lies in the fact, that it is applicable only to patients without history of structural heart disease ${ }^{7}$. Sarasin et al. ${ }^{12}$ identified three predictors of fatal arrhythmias: abnormal ECG, history of congestive heart failure and age above 65 . The only other existing syncope risk stratification point score not included in our study is the San Francisco Syncope Rule ${ }^{8}$, which predicts not only mortality but also serious morbidity. It is not included here due to its low overall sensitivity.

The purpose of this paper is to introduce the above mentioned scores into the Czech medical environment. We further (retrospectively) evaluate the three risk scores
(EGSYS-U, EGSYS-M, OESIL) for patients admitted to the Faculty Hospital in Ostrava and compare the ability of the score systems to classify cardiac syncopes. We evaluate the score systems with respect to their specificity (and sensitivity) and ability to reduce unnecessary hospital admissions.

\section{MATERIALS AND METHODS}

Data origin. Retrospectively, the data from all patients admitted to the University Hospital in Ostrava in the year 2008 (6342) were collected. The data were obtained from electronically stored admission and dismissal protocols of the hospital database - Carecenter. All patients with preliminary diagnosis of syncope (177) were selected, 13 patients were excluded due to dismissal of the original diagnosis, and 11 patients were excluded due to syncope of unknown origin or lack of information relevant for scoring. The remaining 153 patients were enrolled for scoring with the EGSYS (Univariate and Multivariate) and OESIL risk scores (see Appendix 1). For all patients included, the cause of their syncope had been determined during their hospitalization, consequently we were able to classify the syncope as cardiac or non-cardiac. Cardiac etiology of a syncope was investigated and treated according to ESC and national guidelines for syncope and specific cardiac disease ( sick sinus syndrome, atrio-ventricular blockade, ventricular arrhytmia, valvular heart disease etc.). During hospitalization no syncope after treatment was observed.

Statistical analysis methods. For comparing the scores of the two groups (cardiac and non-cardiac), the KruskalWallis test was used. The test compares the medians of the samples, and returns the p-value for the null hypothesis that all samples are drawn from the same population (or equivalently, from different populations with the same distribution). The Kruskal-Wallis test is a non-parametric version of the classical one-way ANOVA, and an extension of the Wilcoxon rank sum test to more than two groups. For comparison of the scores, Spearman's rank correlation was used. A probability below 5\% was considered statistically insignificant.

\section{RESULTS AND DISCUSSION}

First, the ability of the three score systems to classify cardiac syncopes was tested. For each score system, a hypothesis was posed that there is no difference in the score between the cardiac and the non-cardiac group. The hypothesis was rejected for all the three score systems (see Fig. 1). All the three p-values were below 0.0001 . Naturally, there is a strong correlation between the three score systems (Spearman correlation coefficient: $r=0.71$ $\mathrm{p}<0.0001$ for EGSYS-U and OESIL, $\mathrm{r}=0.88 \mathrm{p}<0.0001$ for EGSYS-U and EGSYS-M, $r=0.60 \mathrm{p}<0.0001$ for EGSYS-M and OESIL). 


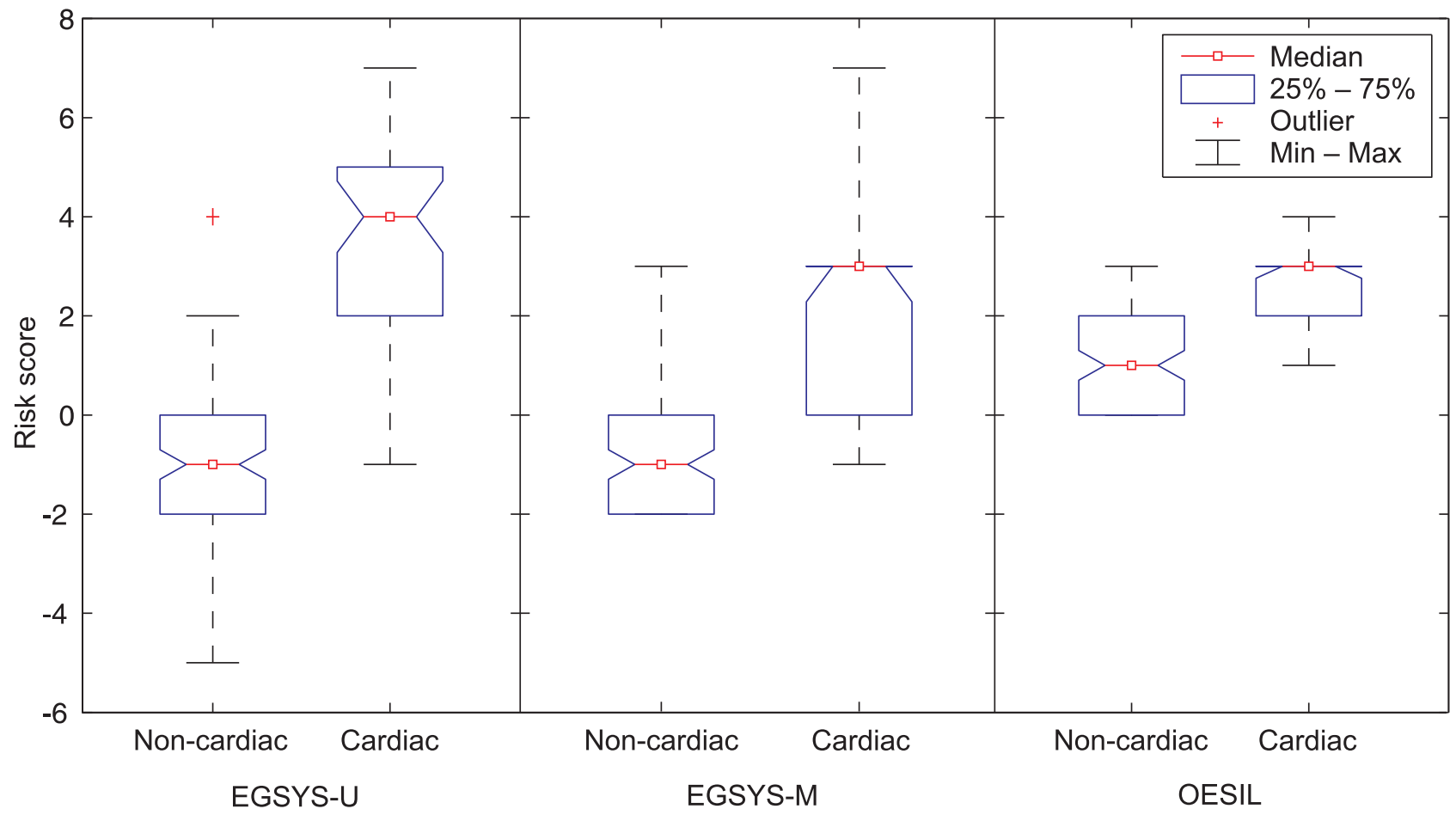

Fig. 1. Comparison of the number of points scored in the three systems by the cardiac and non-cardiac syncope groups.

It is obvious that all the three scores are able to classify cardiac and non-cardiac syncopes and therefore can be used for quick decision making. It would be interesting to see which of the scores has the lowest p-value. However, the p-values in all the three cases is below 0.0001 and their comparison is thus unavailable. Nevertheless, in our setting, we consider the EGSYS-U risk score to be the best option for classification of the cardiac syncope because the quartiles of cardiac syncope scores are furthest apart from the non-cardiac syncope scores (see Fig. 1). This means that (at an appropriate cut-off level) this score sys- tem exhibits both high sensitivity and high specificity (see Table 1 and the discussion below).

For each of the score systems, sensitivity and specificity was evaluated in the following way: Denote a particular value of the score by $x$. The number of patients with cardiac (resp. non-cardiac) syncopes scoring at least $x$ points is denoted by $\mathrm{T}^{+}\left(\mathrm{resp} . \mathrm{T}^{-}\right)$. The total number of patients with cardiac (resp. non-cardiac) syncopes is denoted $\mathrm{D}^{+}$ (resp. $\mathrm{D}^{-}$). The ratio $\mathrm{T}^{+} / \mathrm{D}^{+}$then estimates the sensitivity of the score system at the cut-off level of $x$. The ratio $\mathrm{T}^{-} / \mathrm{D}^{-}$estimates the specificity at the same cut-off level.

Table 1. Sensitivity and specificity of the EGSYS-U, EGSYS-M and OESIL risk scores. $\mathrm{D}^{+}=43$ (true cardiac syncopes), $\mathrm{D}^{-}=110$ (true non-cardiac syncopes). The number of patients with cardiac (resp. non-cardiac) syncopes scoring at least $x$ points (first column) is denoted by $\mathrm{T}^{+}\left(\right.$resp. $\left.\mathrm{T}^{-}\right)$.

\begin{tabular}{|c|c|c|c|c|c|c|c|c|c|c|c|c|}
\hline & \multicolumn{4}{|c|}{ EGSYS-U } & \multicolumn{4}{|c|}{ EGSYS-M } & \multicolumn{4}{|c|}{ OESIL } \\
\hline \begin{tabular}{|c|} 
Score \\
greater or \\
equal to
\end{tabular} & $\mathrm{T}^{+}$ & $\begin{array}{c}\mathrm{T}^{+} / \mathrm{D}^{+} \\
\text {Sensitivity }\end{array}$ & $\mathrm{T}^{-}$ & $\begin{array}{c}\mathrm{T}^{-} / \mathrm{D}^{-} \\
\text {Specificity }\end{array}$ & $\mathrm{T}^{+}$ & $\begin{array}{c}\mathrm{T}^{+} / \mathrm{D}^{+} \\
\text {Sensitivity }\end{array}$ & $\mathrm{T}^{-}$ & $\begin{array}{c}\mathrm{T}^{-} / \mathrm{D}^{-} \\
\text {Specificity }\end{array}$ & $\mathrm{T}^{+}$ & $\begin{array}{c}\mathrm{T}^{+} / \mathrm{D}^{+} \\
\text {Sensitivity }\end{array}$ & $\mathrm{T}^{-}$ & $\begin{array}{c}\mathrm{T}^{-} / \mathrm{D}^{-} \\
\text {Specificity }\end{array}$ \\
\hline 7 & 2 & 0.0465 & 110 & 1 & 1 & 0.0233 & 110 & 1 & & & & \\
\hline 6 & 4 & 0.0930 & 110 & 1 & 2 & 0.0465 & 110 & 1 & & & & \\
\hline 5 & 14 & 0.3256 & 110 & 1 & 3 & 0.0698 & 110 & 1 & & & & \\
\hline 4 & 25 & 0.5814 & 106 & 0.9636 & 3 & 0.0698 & 110 & 1 & 5 & 0.1163 & 110 & 1 \\
\hline 3 & 29 & 0.6744 & 106 & 0.9636 & 24 & 0.5581 & 107 & 0.9727 & 25 & 0.5814 & 105 & 0.9545 \\
\hline 2 & 40 & 0.9302 & 100 & 0.9091 & 28 & 0.6512 & 104 & 0.9455 & 40 & 0.9302 & 60 & 0.5455 \\
\hline 1 & 40 & 0.9302 & 90 & 0.8182 & 28 & 0.6512 & 100 & 0.9091 & 43 & 1 & 31 & 0.2818 \\
\hline $\mathbf{0}$ & 41 & 0.9535 & 79 & 0.7182 & 38 & 0.8837 & 79 & 0.7182 & 43 & 1 & 0 & 0 \\
\hline-1 & 43 & 1 & 50 & 0.4545 & 43 & 1 & 29 & 0.2636 & & & & \\
\hline-2 & 43 & 1 & 25 & 0.2273 & 43 & 1 & 0 & 0 & & & & \\
\hline-3 & 43 & 1 & 9 & 0.0818 & & & & & & & & \\
\hline-4 & 43 & 1 & 1 & 0.0091 & & & & & & & & \\
\hline-5 & 43 & 1 & 0 & 0 & & & & & & & & \\
\hline
\end{tabular}



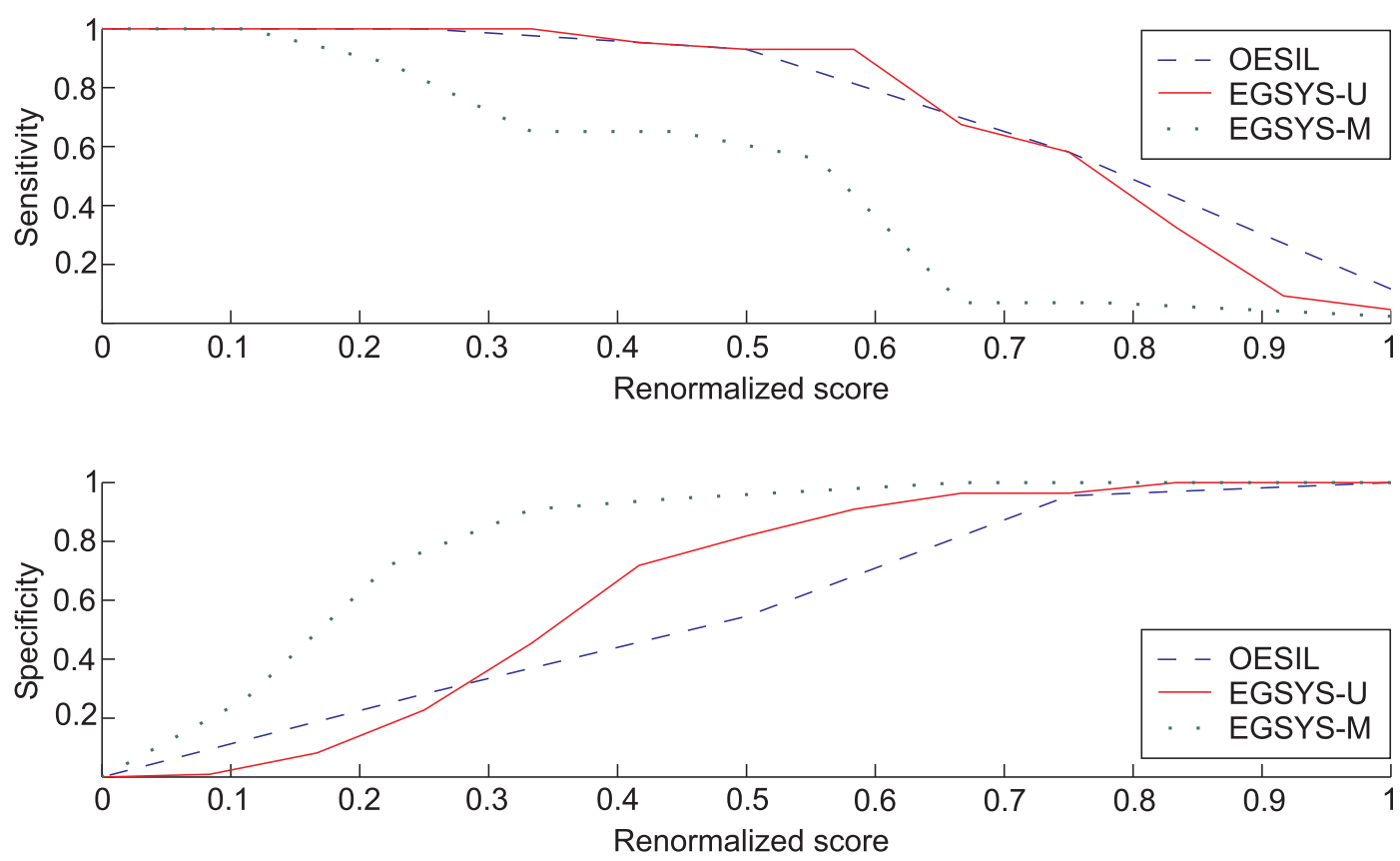

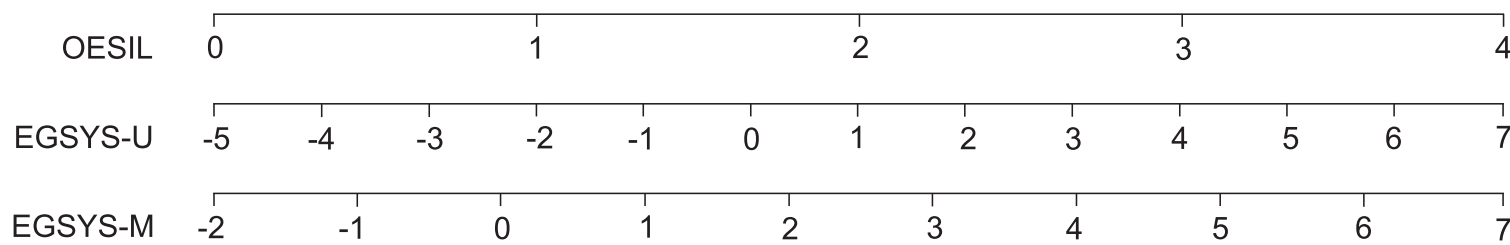

Fig. 2. Sensitivity and specificity of EGSYS and OESIL. The horizontal axis is re-scaled so that 0 represents the minimum possible score and 1 the maximum. The spacing is equidistant. The three scales below the graph show the true scores.

Table 1 shows the results. The sensitivity and specificity of all the three score systems is shown in Fig. 2. Note that the horizontal axis was re-scaled between 0 and 1 , so that the three systems are comparable.

Table 1 may be used for optimizing the hospital admission procedure. If a hospital decides that it must admit e.g. at least $90 \%$ of patients who have undergone a cardiac syncope (i.e. specificity of $90 \%$ is prescribed), according to Table 1, patients with EGSYS-U score below 2 need not be hospitalized. Therefore, one cannot provide an optimal cut-off level (threshold) for the score, unless external criteria are specified. The specification of the criteria is not a matter of statistical analysis of the data but rather a 'political' decision of the hospital.

Most scoring systems (except for the EGSYS) overestimate the importance of age as a predictor of a serious syncope. On the other hand, approximately $46 \%$ of older patients suffering from a heart disease experience a neurally mediated syncope ${ }^{13}$. According to the EGSYS founders ${ }^{10}$, this score is capable of identifying younger high-risk patients by including an effort syncope as a predictor, in spite of a normal ECG and an absence of structural heart disease.

Limitations of the study. It should be emphasized that this is a retrospective cohort study. There is a bias in the data selection, due to the fact that certain patients with syn- cope may not have been hospitalized (and therefore are not included in the study). Consequently, the analyzed population is likely to contain more cases of cardiac syncopes (which appear to be more severe) than the average ED (syncope) population. Moreover, patients with unexplained syncope were excluded from the study.

\section{CONCLUSION}

As already stated, the point of ED decision-making strategy is to evaluate the risk of discharging a patient who has undergone a syncope and treating him/her in outpatients. In the Czech Republic, there are not enough financial resources to establish and maintain SMUs which have been shown to be highly effective in initial syncope evaluation. In this study, we confirmed the original results ${ }^{3,6,10}$ that simple anamnestic data and ECG records organized in the form of a point score can help to recognize cardiac syncopes, which are considered to be the most dangerous ${ }^{10}$. From the three evaluated scores (EGSYS-U, EGSYS-M, and OESIL), we recommend that EGSYS-U be used in ED syncope management, because (at the appropriate cut-off level) it appears to have both high sensitivity and high specificity. E.g. at the cutoff level of 2 points (which is one point below the threshold recommended ${ }^{10}$ ), the sensitivity (cardiac syncopes correctly identified by the test) reaches $93 \%$ while the specificity (non- 
cardiac syncopes correctly identified by the test) stays as high as $91 \%$. However, we should remain aware of the bias in the selection of the patients (see Limitations of the study). The price one has to pay for the best performance of the EGSYS-U test is its complexity - out of the three scoring systems, this is the most extensive one (see Appendix 1). Yet, we believe that the time taken to complete any of the three questionnaires in Appendix 1 is negligible and makes no difference at all. Since there are no cardiologists or syncope management experts working at most of the EDs, simple decision-making guidelines are of great importance. We believe that the above described scoring systems (together with our recommendations based on retrospective evaluation and statistical analysis) can be used to reduce unnecessary hospital admissions and improve syncope risk evaluation.

\section{APPENDIX 1}

\section{EGSYS Risk Score - Univariate (EGSYS-U) ${ }^{9}$}

The point score is found as the sum of the following risk factors:

- Abnormal ECG / Cardiopathy:

- Palpitations / dyspnea:

- Syncope in supine position / Effort syncope:

- Age > 64 years:

- No precipitating and predisposive factors:

- No prodromes:

- Blurred vision:

- Neurovegetative signs during recovery phase:

- Precipitating and predisposive factors:

- Neurovegetative prodromes:

A score greater or equal to 1 implies an increased risk of cardiac syncope, a score less than -2 implies an increased risk of non-cardiac syncope.

\section{EGSYS Risk Score - Multivariate (EGSYS-M $)^{9}$}

The point score is found as the sum of the following risk factors:

- Palpitations:

- Abnormal ECG / Cardiopathy:

- Effort syncope:

- Syncope in supine position:

- Neurovegetative prodromes:

- Precipitating and predisposive factors:

A score greater than 2 implies an increased risk of cardiac syncope.
OESIL risk score ${ }^{6}$

The point score is found as the sum of the following risk factors:

- Age > 65 years:

- History of CV disease:

- Syncope without prodromes:

- Abnormal ECG:

A score greater or equal to 2 implies an increased risk of cardiac death.

\section{REFERENCES}

1. Brignole M, Alboni P, Bendit DG, Bergfeldt L, Blanc JJ, Bloch Thomsen PE et al. Task Force on Syncope, European Society of Cardiology. Guidelines on management (diagnosis and treatment) of syncope, Update 2004. Europace 2004; 15: 35-39.

2. Soteriades ES, Evans JC, Larson MG, Chen MH, Chen L, Benjamin EJ et al. Incidence and prognosis of syncope. N Engl J Med 2002; 347: 878-885.

3. Brignole M, Disertoni M, Menozzi C, Raviele A, Alboni P, Pitzalis MV et al. Evaluation of Guidelines in Syncope Study (EGSYS) group. Management of syncope referred urgently to general hospitals with and without syncope units. Europace 2003; 5: 293-298.

4. Kenny RA, O'Shea D, Walker HF. Impact of a dedicated syncope and falls facility for older adults on emergency beds. Age Ageing 2002; 31: 272-275.

5. Shen WK, Decker WW, Smars PA, Goyal DG, Walker AE, Hodge DO et al. Syncope Evaluation in the Emergency Department Study (SEEDS): A multidisciplinary approach to syncope management. Circulation 2004; 14: 3636-3645.

6. Colivicchi F, Ammirati F, Melina D, Guido V, Imperoli G, Santini $\mathrm{M}$ et al. OESIL (Osservatorio Epidemiologico sulla Sincope nel Lazio) Study Investigators. Development and prospective validation of risk stratification system for patients with syncope in the emergency department: the OESIL risk score. Eur Heart J 2003; 24: 811-819.

7. Sheldon R, Rose S, Connolly S, Ritchie D, Koshman ML, Frenneaux $\mathrm{M}$ et al. Diagnostic criteria for vasovagal syncope based on a quantitative history. Eur Heart J 2006; 27: 344-350.

8. Quinn JV, Stiell IG, McDermott DA, Sellers KL, Kohn MA, Wells GA. Derivation of the San Francisco Syncope Rule to predict patiens with short-term serious outcomes. Ann Emerg Med 2004; 43: 224-232.

9. Brignole M et al. Syncope Unit Project. 2006 Nov available from http://www.aiac.it/upload/documenti/redazione_medici/ DOC20061111 9614421.doc [cited 2009, June 15],[about 17 p.]

10. Del Rosso A, Ungar A, Maggi R, Giada F, Petix NR, De Santo T, et al. Clinical predictors of cardiac syncope at initial evaluation in patients referred urgently to a general hospital: the EGSYS score. Heart 2008; 94: 1620-1626.

11. Calkins H, Shyr Y, Frumin H, Schork A, Morady F. The value of the clinical history in the differentiation of syncope due to ventricular tachycardia, atrioventricular block and neurocardiogenic syncope. Am J Med 1995; 98: 365-373.

12. Sarasin FP, Hanusa BH, Perneger T, Louis-Simonet M, Rajeswaran A, Kapoor WN. A risk score to predict arrhytmias in patients with unexplained syncope. Acad Emerg Med 2003; 10: 1312-1317.

13. Del Rosso A, Alboni P, Brignole M, Menozzi C, Raviele A. Relation of clinical presentation of syncope to the age of patients. Am J Cardiol 2005; 96: 1431-1435. 